\title{
GUEST EDITORIAL Special Issue: Design computing and cognition
}

\author{
ANDY DONG \\ Key Centre of Design Computing and Cognition, University of Sydney, Australia
}

\begin{abstract}
The field of research in design computing and cognition focuses on computational theories and systems that enact design. Design computing and cognition produces a unifying framework to model and explain design beyond the description of "design computing and cognition," as in "design computing" and "design cognition" as two cognate disciplines. Research in design computing and cognition recognizes not only the essential relationship between human cognitive processes as models of computation but also how models of computation inspire conceptual realizations of human cognition in design. The articles in this Special Issue address the concomitant key areas of research in design computing and cognition: computational models of design, computational representations in design, computational design systems, and design cognition. The computationally inspired perspectives, metaphors, models, and theories that the papers deliver create a base for computing and cognition to (re)shape design practice and its role in design science and inquiry.
\end{abstract}

The field of research in design computing and cognition focuses on computational theories and systems that enact design. The theme of "design computing and cognition" could be interpreted as "design computing" and "design cognition," as two terms merged, as two cognate disciplines described within a single phrase. Rather, design computing and cognition interpreted as a unifying framework models and explains the practice of design and establishes a community of research by recognizing not only the essential relationship between human cognitive processes as models of computation but also how models of computation inspire conceptual realizations of human cognition in design.

The five papers selected for publication in this Special Issue exemplify this approach to design research and deal with the primary concerns of research in design computing and cognition: computational models of design, computational representations in design, computational design systems, and design cognition.

The first paper, by Sosa and Gero, presents a novel approach to computational models of design through a computational exploration of creativity as a social phenom-

Reprint requests to: Andy Dong, Key Centre of Design Computing and Cognition, University of Sydney, Wilkinson Building (G04), Sydney, NSW 2006, Australia. E-mail: adon3656@mail.usyd.edu.au enon. Through the simulation of designers as (computational) agents in a society, the authors explore how social situations and structures regulate the production and evaluation of creative ideas. In contrast to individuated computational models of creativity, the authors' social framework demonstrates that "creative situations" organize how designers behave in a society, and that a designer's creativity is in part framed by the "structures of authority in creativity and innovation."

The next two papers examine the computational representations of shapes in design. The papers adopt two approaches to the general problem of recognizing the information contained in shapes. Ostrosi and Ferney present a five-phase, grammar-based approach for recognizing canonical features or features in interaction. The authors present a set of production rules that parse graphs based on their feature grammar and demonstrate the technique on a representative shape. Krstic develops formal decompositions of shapes within an algebraic taxonomy. In pushing the boundaries of the shape decomposition algebras, Krstic opens the potential to model formally the generation of shapes by designers.

The next paper by de Vries et al. recognizes that words have been an underemployed source of information for designers. They developed word graphs to stimulate architects' thinking during design through linguistic rather than visual forms. What is perhaps most intriguing about word 
graphs is how the interactions with the semantic representations and relationships between words can serve equivalent functions as graphical modeling in mediating interaction between the design representations and the architects' cognitive structures.

Finally, the paper by Heylighen and Martin proposes a connectionist model to analyze the emergence and development of concepts from the viewpoint of the design process rather than as a post hoc recollection. Like the study by Coyne et al. (1993), the authors propose that connectionists models account for the restless nature of concepts during design. The twist to the paper is that the authors ultimately question whether a suitable model, cognitive or computational, of a "concept" exists. Couching concepts in systemic and structural terms may significantly miss the "becoming" of a concept. Perhaps, as the paper suggests, the concept of a concept is dissolved in movement. The essence of a concept appears in the dynamics of activation of the "concept units" and "feature units."

The authors in this Special Issue originally presented their papers at the First International Conference on Design Computing and Cognition (DCC'04) that convened at MIT on July 19-21, 2004. Sponsored by the Key Centre of Design Computing and Cognition at the University of Sydney and the School of Architecture and Planning at MIT, the conference brought together a forum for the discussion of research ranging from agents in design to computational theories applied to intelligent facilities management. With DCC'06 set to take place July 10-12, 2006, at the Technical University of Eindhoven in The Netherlands, we look forward to another stimulating conference presenting the latest research in design computing and cognition.
Authors presenting at DCC'04 were invited to submit extended and revised versions of their original contributions, which had already been independently reviewed by three readers, for this Special Issue. The papers selected for presentation in this Special Issue were additionally reviewed by at least two new independent reviewers and then revised, incorporating the referees' comments. The editorial team thanks all of the authors from DCC'04 who contributed their papers to this Special Issue and the authors whose papers appear in this Special Issue for their significant effort in revising their manuscripts. Special thanks are extended to the referees for giving their valuable comments on the papers.

Finally, the Guest Editor expresses thanks to Professor David Brown, Editor in Chief of AIEDAM, for his advice during the process of editing this Special Issue.

\section{REFERENCES}

Coyne, R.D., Newton, S., \& Sudweeks, F. (1993). A connectionist view of creative design reasoning. In Modeling Creativity and KnowledgeBased Creative Design (Gero, J.S., \& Maher, M.L., Eds.), pp. 177209. Hillsdale, NJ: Erlbaum.

Andy Dong is a Lecturer in design computing and cognition at the University of Sydney. His research focuses on describing and modeling design practice using computational linguistics and developing related pervasive computing systems that assist designers in aggregating, accumulating, and appraising the projected realities deployed in their system of design lexis. 